\title{
SEARCHING THE CONTINUUM FOR PRIMEVAL GALAXIES
}

\author{
MARSHALL L. MCCALL AND MICHAEL M. DEROBERTIS \\ York University, Department of Physics and Astronomy \\ 4700 Keele St., North York, Ontario, Canada M3J 1P3
}

A population of primeval galaxies (PG's) should be detectable by directly imaging with two intermediate-band filters tuned to either side of the Lyman break (DeRobertis, M. M., and McCall, M. L. 1995, A.J., 109, 1947). In the figure below, the solid and short-dashed curves show the flux (left scale) as a function of redshift from a PG 0.7 Gyr old with a total stellar mass of $5 \times 10^{10} \mathcal{M}_{\odot}$ as seen through filters with rest-frame passbands $890 \pm 30 \AA$ (' $\beta$ ') and $1010 \pm 30 \AA$ (' $\rho$ '), respectively, moved to redshift 5 . The upper curves depict the colour $\beta-\rho$ (right scale); the dotted line is for the $0.7 \mathrm{Gyr}$ population, and the dot-dashed line is for a $7.5 \mathrm{Gyr}$ model. A source can be identified as a PG if it can be clearly detected in the $\rho$ filter and if it has a colour greater than +0.75 mag. Confusion with any old stellar systems at lower redshifts can be eliminated by supplementing observations with Gunn $r$ and $i$. The colour condition selects Lyman break objects between redshifts 4.7 and 5.4, a range over an order of magnitude greater than is achievable through an emission line survey. The discriminatory power of the technique is not affected by internal dust.

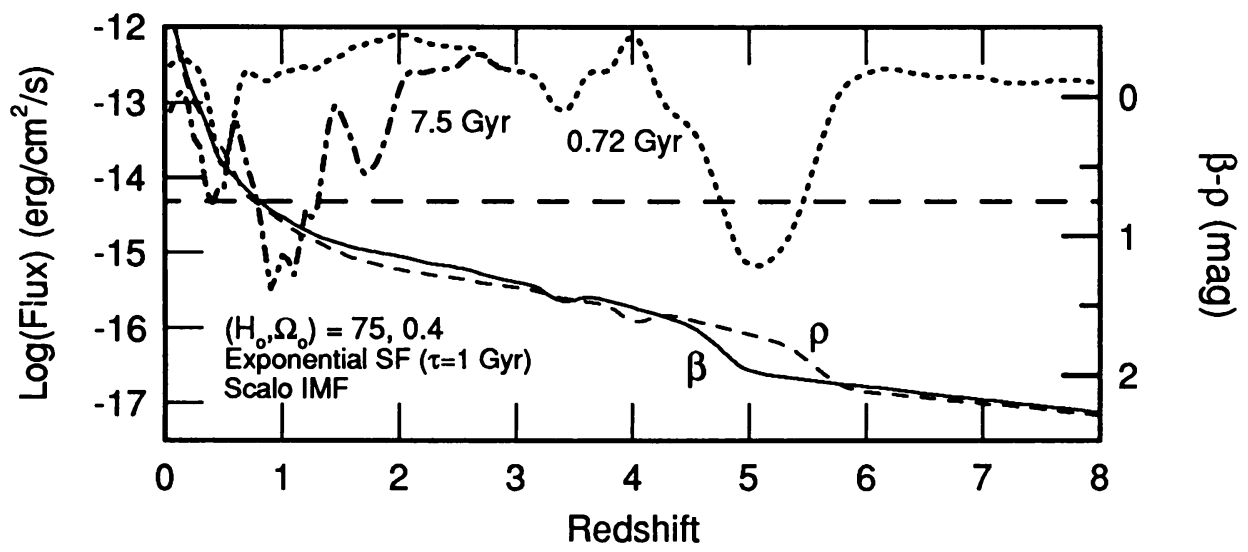

